\title{
45. SONIC VELOCITIES AND DENSITIES OF BASALTS FROM THE NAZCA PLATE, DSDP LEG 34
}

\author{
Matthew H. Salisbury ${ }^{1}$ and Nikolas I. Christensen, \\ Department of Geological Sciences, University of Washington, Seattle, Washington
}

\section{INTRODUCTION}

Compressional $(\mathrm{Vp})$ and shear $(\mathrm{Vs})$ wave velocities have been measured in the laboratory under conditions of hydrostatic confining pressure to $6.0 \mathrm{kbar}$ through 15 samples of basalt recovered during DSDP Leg 34 from Sites 319,320 , and 321 on the Nazca plate. All velocities were determined using the pulse transmission technique through water-saturated samples maintained at pore pressures much lower than confining pressure.

The purpose of this investigation is to determine the velocities and elastic properties of samples from the upper levels of layer 2 in the Nazca plate under conditions of confining pressure and water saturation appropriate to the sea floor for eventual comparison with refraction data, and to determine from thin section analysis the causes of variations among these properties from site to site and with depth at any given site.

Basalts recovered from Holes 319 and 319A, the youngest site drilled on Leg 34 (14 m.y.), are relatively fresh, but vary irregularly in grain size, texture, and degree of alteration with depth. The shallowest samples, 319-13-1, 52-55 cm and 319A-1-1, 32-35 cm, consist, respectively, of fine-grained basalt with variolitic texture and medium- to coarse-grained basalt with variolitic to subophitic texture. Both display minimal alteration. Samples 319A-2-3, 46-48 cm; 319A-3-2, 114-117 cm; and $319 \mathrm{~A}-3-4,85-88 \mathrm{~cm}$, listed in order of increasing depth, consist of medium-grained diabase with ophitic texture. The two uppermost diabase samples display minor alteration decreasing with depth. The lowermost diabase examined, Sample 319A-14-1, 137-140 cm, displays a fine-grained subophitic texture with minor alteration. All of the diabase samples, except 319A-3-4, 85-88 cm, contain a small number of bent pyroxene grains. Sample $319 \mathrm{~A}-5-1,80-83 \mathrm{~cm}$, immediately below the diabases, consists of fresh, coarse-grained basalt with subvariolitic to subophitic texture. The two deepest samples examined, 319A-6-1, 145-146 cm and 319A-7-1, 65-68 cm, consist, respectively, of fine- to medium-grained basalt with trachytic, subvariolitic to intersertal texture, and coarse-grained basalt with subvariolitic to subophitic texture. Both are slightly altered, with alteration increasing downward.

The basalts recovered from Hole 320B (28 m.y.) are uniformly fine grained, but vary in texture and degree of alteration with depth. The uppermost sample, 320B-3-1, 64-67 cm, is an altered, microporphyritic, vesicular

'Currently at Department of Geological Sciences, State University of New York, Binghampton, New York. basalt with an intersertal texture. Sample 320B-4-1, 144 $147 \mathrm{~cm}$ is variolitic in texture and is only partially altered. The deepest sample studied from this hole, 320B-5, CC, is composed of a fresh basalt, again, of variolitic texture.

The basalts from Site 321, the oldest site drilled on Leg 34 (40 m.y.), increase in grain size from fine to coarse grained with depth and are uniformly intergranular in texture. Alteration varies irregularly with depth; the shallowest sample examined, 321-13-4, 104$107 \mathrm{~cm}$, is an altered basalt, whereas Sample 321-14-1, $76-79 \mathrm{~cm}$ and the deepest sample, $321-14-4,51-54 \mathrm{~cm}$, are, respectively, fresh and only partially altered. Vesicles, present in Samples 321-14-1, 76-79 cm and 321$14-4,51-54 \mathrm{~cm}$ are filled with calcite and nontronite, respectively.

\section{VELOCITIES, DENSITIES, AND ELASTIC MODULI}

Velocities were measured through samples cut in the form of right circular cylinders $2.54 \mathrm{~cm}$ in diameter and 2 to $4 \mathrm{~cm}$ in length. The velocities, which are given in Table 1, were measured using the pulse transmission technique described in detail by Birch (1960). Compressional and shear waves were generated, respectively, by means of 1 to $2 \mathrm{mHz}$ barium titanate and AC-cut quartz transducers. All samples were water saturated prior to velocity measurement; during the runs pore pressures were maintained at values much less than confining pressures by placing 100-mesh screens between the samples and copper jackets. The densities reported in Table 1 were calculated from the mass and measured dimensions of the cores used for velocity measurement.

Values of the compressional to shear wave velocity ratio $(\mathrm{Vp} / \mathrm{Vs})$, Poisson's ratio $(\sigma)$, the seismic parameter $(\phi)$, the bulk modulus $(\kappa)$, the compressibility $(\beta)$, the shear modulus $(\mu)$, Young's modulus (E), and Lame's constant $(\lambda)$ calculated from measured densities and velocities are given in Table 2 for each sample at selected pressures.

\section{DISCUSSION}

Basalts recovered from the upper levels of layer 2 during the Deep Sea Drilling Project have been observed (Christensen et al., 1974b) to range widely in compressional and shear wave velocity $(3.4-6.6 \mathrm{~km} / \mathrm{sec}$ for $\mathrm{Vp}$ and 1.7-3.6 km/sec for Vs at $0.5 \mathrm{kbar}$ ). As can be seen in Figure 1, however, the samples examined from Leg 34 are unusual in exhibiting an uncommonly high and narrow range of velocities $(5.2-6.3 \mathrm{~km} / \mathrm{sec}$ for $\mathrm{Vp}$ and $2.8-3.5 \mathrm{~km} / \mathrm{sec}$ for $\mathrm{Vs})$. Since none of the sites on Leg 34 are more than 40 m.y. old, the high velocities 
TABLE 1

Compressional (P) and Shear (S) Wave Velocities

\begin{tabular}{|c|c|c|c|c|c|c|c|c|c|c|}
\hline \multirow{2}{*}{$\begin{array}{c}\text { Sample } \\
\text { (Interval in } \mathrm{cm} \text { ) }\end{array}$} & \multirow{2}{*}{$\begin{array}{c}\text { Bulk } \\
\text { Density }\end{array}$} & \multirow[b]{2}{*}{ Mode } & \multicolumn{8}{|c|}{ Velocity $(\mathrm{km} / \mathrm{sec})$ at Varying Pressures $(\mathrm{kb})$} \\
\hline & & & 0.2 & 0.4 & 0.6 & 0.8 & 1.0 & 2.0 & 4.0 & 6.0 \\
\hline $\begin{array}{l}319 A-1-1 \\
32-35\end{array}$ & $\begin{array}{l}2.915 \\
2.915\end{array}$ & $\begin{array}{l}P \\
S\end{array}$ & $\begin{array}{l}6.06 \\
3.32\end{array}$ & $\begin{array}{l}6.11 \\
3.33\end{array}$ & $\begin{array}{l}6.14 \\
3.34\end{array}$ & $\begin{array}{l}6.17 \\
3.35\end{array}$ & $\begin{array}{l}6.20 \\
3.36\end{array}$ & $\begin{array}{l}6.28 \\
3.40\end{array}$ & $\begin{array}{l}6.38 \\
3.44\end{array}$ & $\begin{array}{l}6.41 \\
3.47\end{array}$ \\
\hline $\begin{array}{l}319 A-2-3 \\
46-48\end{array}$ & $\begin{array}{l}2.864 \\
2.864\end{array}$ & $\begin{array}{l}\mathrm{P} \\
\mathrm{S}\end{array}$ & $\begin{array}{l}5.92 \\
3.19\end{array}$ & $\begin{array}{l}5.94 \\
3.20\end{array}$ & $\begin{array}{l}5.95 \\
3.22\end{array}$ & $\begin{array}{l}5.98 \\
3.23\end{array}$ & $\begin{array}{l}6.00 \\
3.24\end{array}$ & $\begin{array}{l}6.06 \\
3.29\end{array}$ & $\begin{array}{l}6.19 \\
3.33\end{array}$ & $\begin{array}{l}6.32 \\
3.35\end{array}$ \\
\hline $\begin{array}{l}319 A-3-2 \\
114-117\end{array}$ & $\begin{array}{l}2.923 \\
2.923\end{array}$ & $\begin{array}{l}\mathrm{P} \\
\mathrm{S}\end{array}$ & $\begin{array}{l}6.06 \\
3.28\end{array}$ & $\begin{array}{l}6.09 \\
3.29\end{array}$ & $\begin{array}{l}6.12 \\
3.32\end{array}$ & $\begin{array}{l}6.15 \\
3.34\end{array}$ & $\begin{array}{l}6.17 \\
3.35\end{array}$ & $\begin{array}{l}6.27 \\
3.39\end{array}$ & $\begin{array}{l}6.38 \\
3.43\end{array}$ & $\begin{array}{l}6.44 \\
3.47\end{array}$ \\
\hline $\begin{array}{l}319 A-3-4 \\
85-88\end{array}$ & $\begin{array}{l}2.939 \\
2.939\end{array}$ & $\begin{array}{l}\mathrm{P} \\
\mathrm{S}\end{array}$ & $\begin{array}{l}6.14 \\
3.36\end{array}$ & $\begin{array}{l}6.19 \\
3.37\end{array}$ & $\begin{array}{l}6.22 \\
3.37\end{array}$ & $\begin{array}{l}6.25 \\
3.38\end{array}$ & $\begin{array}{l}6.27 \\
3.39\end{array}$ & $\begin{array}{l}6.34 \\
3.41\end{array}$ & $\begin{array}{l}6.43 \\
3.45\end{array}$ & $\begin{array}{l}6.47 \\
3.46\end{array}$ \\
\hline $\begin{array}{l}319 A-4-1, \\
137-140\end{array}$ & $\begin{array}{l}2.911 \\
2.911\end{array}$ & $\begin{array}{l}\mathbf{P} \\
\mathrm{S}\end{array}$ & $\begin{array}{l}6.00 \\
3.30\end{array}$ & $\begin{array}{l}6.03 \\
3.31\end{array}$ & $\begin{array}{l}6.06 \\
3.32\end{array}$ & $\begin{array}{l}6.08 \\
3.33\end{array}$ & $\begin{array}{l}6.09 \\
3.33\end{array}$ & $\begin{array}{l}6.15 \\
3.36\end{array}$ & $\begin{array}{l}6.23 \\
3.39\end{array}$ & $\begin{array}{l}6.31 \\
3.40\end{array}$ \\
\hline $\begin{array}{l}319 A-5-1, \\
80-83\end{array}$ & $\begin{array}{l}2.948 \\
2.948\end{array}$ & $\begin{array}{l}\mathbf{P} \\
\mathrm{S}\end{array}$ & $\begin{array}{l}6.24 \\
3.49\end{array}$ & $\begin{array}{l}6.28 \\
3.49\end{array}$ & $\begin{array}{l}6.30 \\
3.50\end{array}$ & $\begin{array}{l}6.32 \\
3.50\end{array}$ & $\begin{array}{l}6.34 \\
3.50\end{array}$ & $\begin{array}{l}6.40 \\
3.52\end{array}$ & $\begin{array}{l}6.46 \\
3.53\end{array}$ & $\begin{array}{l}6.49 \\
3.53\end{array}$ \\
\hline $\begin{array}{l}319 A-6-1 \\
145-148\end{array}$ & $\begin{array}{l}2.882 \\
2.882\end{array}$ & $\begin{array}{l}\mathrm{P} \\
\mathrm{S}\end{array}$ & $\begin{array}{l}6.11 \\
3.27\end{array}$ & $\begin{array}{l}6.12 \\
3.29\end{array}$ & $\begin{array}{l}6.14 \\
3.31\end{array}$ & $\begin{array}{l}6.15 \\
3.32\end{array}$ & $\begin{array}{l}6.16 \\
3.33\end{array}$ & $\begin{array}{l}6.19 \\
3.35\end{array}$ & $\begin{array}{l}6.23 \\
3.35\end{array}$ & $\begin{array}{l}6.26 \\
3.36\end{array}$ \\
\hline $\begin{array}{l}319 A-7-1, \\
65-68\end{array}$ & $\begin{array}{l}2.851 \\
2.851\end{array}$ & $\begin{array}{l}P \\
S\end{array}$ & $\begin{array}{l}5.93 \\
3.24\end{array}$ & $\begin{array}{l}5.97 \\
3.25\end{array}$ & $\begin{array}{l}6.00 \\
3.26\end{array}$ & $\begin{array}{l}6.01 \\
3.27\end{array}$ & $\begin{array}{l}6.03 \\
3.28\end{array}$ & $\begin{array}{l}6.07 \\
3.30\end{array}$ & $\begin{array}{l}6.13 \\
3.33\end{array}$ & $\begin{array}{l}6.18 \\
3.35\end{array}$ \\
\hline $\begin{array}{l}319-13-1, \\
52-55\end{array}$ & $\begin{array}{l}2.920 \\
2.920\end{array}$ & $\begin{array}{l}\mathrm{P} \\
\mathrm{S}\end{array}$ & $\begin{array}{l}6.13 \\
3.26\end{array}$ & $\begin{array}{l}6.17 \\
3.29\end{array}$ & $\begin{array}{l}6.19 \\
3.32\end{array}$ & $\begin{array}{l}6.21 \\
3.34\end{array}$ & $\begin{array}{l}6.23 \\
3.36\end{array}$ & $\begin{array}{l}6.30 \\
3.41\end{array}$ & $\begin{array}{l}6.38 \\
3.45\end{array}$ & $\begin{array}{l}6.46 \\
3.46\end{array}$ \\
\hline $\begin{array}{l}\text { 320B-3-1, } \\
64-67\end{array}$ & $\begin{array}{l}2.725 \\
2.725\end{array}$ & $\begin{array}{l}\mathrm{P} \\
\mathrm{S}\end{array}$ & $\begin{array}{l}5.25 \\
2.85\end{array}$ & $\begin{array}{l}5.27 \\
2.86\end{array}$ & $\begin{array}{l}5.28 \\
2.88\end{array}$ & $\begin{array}{l}5.29 \\
2.89\end{array}$ & $\begin{array}{l}5.31 \\
2.89\end{array}$ & $\begin{array}{l}5.37 \\
2.93\end{array}$ & $\begin{array}{l}5.48 \\
2.97\end{array}$ & $\begin{array}{l}5.61 \\
3.00\end{array}$ \\
\hline $\begin{array}{l}320 \mathrm{~B}-4-1 \\
144-147\end{array}$ & $\begin{array}{l}2.837 \\
2.837\end{array}$ & $\begin{array}{l}\mathrm{P} \\
\mathrm{S}\end{array}$ & $\begin{array}{l}5.62 \\
3.01\end{array}$ & $\begin{array}{l}5.66 \\
3.03\end{array}$ & $\begin{array}{l}5.71 \\
3.06\end{array}$ & $\begin{array}{l}5.74 \\
3.08\end{array}$ & $\begin{array}{l}5.77 \\
3.10\end{array}$ & $\begin{array}{l}5.89 \\
3.16\end{array}$ & $\begin{array}{l}6.03 \\
3.20\end{array}$ & $\begin{array}{l}6.14 \\
3.25\end{array}$ \\
\hline $320 \mathrm{~B}, 5, \mathrm{CC}$ & $\begin{array}{l}2.832 \\
2.832\end{array}$ & $\begin{array}{l}\mathrm{P} \\
\mathrm{S}\end{array}$ & $\begin{array}{l}6.01 \\
3.24\end{array}$ & $\begin{array}{l}6.04 \\
3.27\end{array}$ & $\begin{array}{l}6.07 \\
3.28\end{array}$ & $\begin{array}{l}6.08 \\
3.30\end{array}$ & $\begin{array}{l}6.10 \\
3.31\end{array}$ & $\begin{array}{l}6.14 \\
3.38\end{array}$ & $\begin{array}{l}6.19 \\
3.44\end{array}$ & $\begin{array}{l}6.24 \\
3.48\end{array}$ \\
\hline $\begin{array}{l}321-13-4 \\
104-107\end{array}$ & $\begin{array}{l}2.822 \\
2.822\end{array}$ & $\begin{array}{l}\mathbf{P} \\
\mathrm{S}\end{array}$ & $\begin{array}{l}5.22 \\
2.84\end{array}$ & $\begin{array}{l}5.26 \\
2.86\end{array}$ & $\begin{array}{l}5.29 \\
2.88\end{array}$ & $\begin{array}{l}5.32 \\
2.89\end{array}$ & $\begin{array}{l}5.35 \\
2.90\end{array}$ & $\begin{array}{l}5.44 \\
2.96\end{array}$ & $\begin{array}{l}5.57 \\
3.02\end{array}$ & $\begin{array}{l}5.70 \\
3.05\end{array}$ \\
\hline $\begin{array}{l}321-14-1 \\
76-79\end{array}$ & $\begin{array}{l}2.900 \\
2.900\end{array}$ & $\begin{array}{l}\mathbf{P} \\
\mathrm{S}\end{array}$ & $\begin{array}{l}6.03 \\
3.28\end{array}$ & $\begin{array}{l}6.05 \\
3.30\end{array}$ & $\begin{array}{l}6.06 \\
3.31\end{array}$ & $\begin{array}{l}6.07 \\
3.33\end{array}$ & $\begin{array}{l}6.08 \\
3.34\end{array}$ & $\begin{array}{l}6.12 \\
3.38\end{array}$ & $\begin{array}{l}6.19 \\
3.42\end{array}$ & $\begin{array}{l}6.26 \\
3.45\end{array}$ \\
\hline $\begin{array}{l}321-14-4 \\
51-54\end{array}$ & $\begin{array}{l}2.915 \\
2.915\end{array}$ & $\begin{array}{l}\mathbf{P} \\
\mathrm{S}\end{array}$ & $\begin{array}{l}5.67 \\
3.09\end{array}$ & $\begin{array}{l}5.71 \\
3.11\end{array}$ & $\begin{array}{l}5.73 \\
3.13\end{array}$ & $\begin{array}{l}5.76 \\
3.14\end{array}$ & $\begin{array}{l}5.78 \\
3.16\end{array}$ & $\begin{array}{l}5.86 \\
3.22\end{array}$ & $\begin{array}{l}5.95 \\
3.29\end{array}$ & $\begin{array}{l}6.00 \\
3.32\end{array}$ \\
\hline
\end{tabular}

observed are not inconsistent with the observations of Christensen and Salisbury $(1972,1973)$ that the seismic velocities of submarine basalts decrease markedly with age in response to progressive submarine weathering.

In this context, it is significant that none of the samples examined in this study appear to be strongly weathered in thin section, but that those samples which display minor weathering and depressed velocities (for example, Samples 320B-3-1, 64-67 cm and 321-13-4, $104-107 \mathrm{~cm}$ ) are from the oldest sites drilled on Leg 34 .

Except perhaps at Site 320B where Vp is observed to increase downward at the rate of $0.04 \mathrm{~km} / \mathrm{sec} / \mathrm{m}$ throughout the 21-meter basement interval sampled, no velocity gradients as convincing as those reconstructed from laboratory studies by Christensen et al. (1974b) for Sites 259 and 261 on Leg 27 are observed at the Leg 34 sites. Minor fluctuations in velocity with depth at the site of deepest basement penetration $(48.5 \mathrm{~m})$ on Leg 34 , Site 319A, reflect changes in grain size, glass content, vesicularity, and weathering associated with successively deeper flow units. Fluctuations of a similar nature are observed at Site 321.

Since little refraction data have been reported to date from the vicinity of the sites drilled on Leg 34, it is difficult to make a direct comparison of the velocities reported in Table 1 and those for the upper levels of layer 2 in the Nazca plate. Since the presence of in- terlayed sediments and pillow basalts in the upper levels of layer 2 may be expected to lower refraction velocities and since the presence of such units is a likely explanation of the difficulties in basement drilling and recovery experienced on Leg 34, the velocities reported in Table 1 probably represent the upper limit of refraction velocities in this region.

\section{ACKNOWLEDGMENTS}

We wish to thank Robert McConaghy and Michael Brown for their assistance in the maintenance and operation of the high pressure system. This investigation was supported by the Office of Naval Research Contract N-00014-67-A-0103-0014 and National Science Foundation Grant GA-36138.

\section{REFERENCES}

Birch, F., 1960. The velocity of compressional waves in rocks to 10 kilobars, 1: J. Geophys. Res., v. 65, p. 1083.

Christensen, 1973a. Compressional and shear wave velocities in basaltic rocks, Deep Sea Drilling Project, Leg 16. In van Andel, T.H., Heath, G.R., et al., Initial Reports of the Deep Sea Drilling Project, Volume 16: Washington (U.S. Government Printing Office), p. 647. 1973b. Compressional and shear wave velocities and elastic moduli of basalts, Deep Sea Drilling Project, Leg 19. In Creager, J.S., Scholl, D.W., et al., Initial Reports of the Deep Sea Drilling Project, Volume 19: Washington (U.S. Government Printing Office), p. 657. 
TABLE 2

Elastic Constants, Leg 34

\begin{tabular}{|c|c|c|c|c|c|c|c|c|c|}
\hline $\begin{array}{c}\text { Sample } \\
\text { (Interval in } \mathrm{cm} \text { ) }\end{array}$ & $\begin{array}{l}\text { Pressure } \\
\quad(\mathrm{kb})\end{array}$ & $\mathrm{V}_{\mathrm{p}} / \mathrm{V}_{\mathrm{s}}$ & $\sigma$ & $\begin{array}{c}\phi \\
(\mathrm{km} / \mathrm{sec})^{2}\end{array}$ & $\begin{array}{c}k \\
(\mathrm{Mb})\end{array}$ & $\left(\begin{array}{c}\beta \\
\left(\mathrm{Mb}^{-1}\right)\end{array}\right.$ & $\begin{array}{c}\mu \\
(\mathrm{Mb})\end{array}$ & $\begin{array}{c}\mathrm{E} \\
(\mathrm{Mb})\end{array}$ & $\begin{array}{c}\lambda \\
(\mathrm{Mb})\end{array}$ \\
\hline $\begin{array}{l}\text { 319A-1-1, } \\
32-35\end{array}$ & $\begin{array}{l}0.4 \\
1.0 \\
2.0 \\
6.0\end{array}$ & $\begin{array}{l}1.83 \\
1.84 \\
1.85 \\
1.84\end{array}$ & $\begin{array}{l}0.29 \\
0.29 \\
0.29 \\
0.29\end{array}$ & $\begin{array}{l}22.5 \\
23.3 \\
24.0 \\
24.9\end{array}$ & $\begin{array}{l}0.66 \\
0.68 \\
0.70 \\
0.73\end{array}$ & $\begin{array}{l}1.53 \\
1.47 \\
1.42 \\
1.37\end{array}$ & $\begin{array}{l}0.32 \\
0.33 \\
0.34 \\
0.35\end{array}$ & $\begin{array}{l}0.83 \\
0.85 \\
0.87 \\
0.91\end{array}$ & $\begin{array}{l}0.44 \\
0.46 \\
0.48 \\
0.50\end{array}$ \\
\hline $\begin{array}{l}319 A-2-3 \\
46-48\end{array}$ & $\begin{array}{l}0.4 \\
1.0 \\
2.0 \\
6.0\end{array}$ & $\begin{array}{l}1.85 \\
1.85 \\
1.84 \\
1.89\end{array}$ & $\begin{array}{l}0.30 \\
0.29 \\
0.29 \\
0.30\end{array}$ & $\begin{array}{l}21.6 \\
21.9 \\
22.3 \\
24.8\end{array}$ & $\begin{array}{l}0.62 \\
0.63 \\
0.64 \\
0.72\end{array}$ & $\begin{array}{l}1.62 \\
1.59 \\
1.56 \\
1.40\end{array}$ & $\begin{array}{l}0.29 \\
0.30 \\
0.31 \\
0.32\end{array}$ & $\begin{array}{l}0.76 \\
0.80 \\
0.80 \\
0.84\end{array}$ & $\begin{array}{l}0.42 \\
0.43 \\
0.43 \\
0.50\end{array}$ \\
\hline $\begin{array}{l}319 A-3-2 \\
114-117\end{array}$ & $\begin{array}{l}0.4 \\
1.0 \\
2.0 \\
6.0\end{array}$ & $\begin{array}{l}1.85 \\
1.84 \\
1.85 \\
1.86\end{array}$ & $\begin{array}{l}0.29 \\
0.29 \\
0.29 \\
0.30\end{array}$ & $\begin{array}{l}22.7 \\
23.1 \\
23.9 \\
25.4\end{array}$ & $\begin{array}{l}0.66 \\
0.68 \\
0.70 \\
0.75\end{array}$ & $\begin{array}{l}1.51 \\
1.48 \\
1.43 \\
1.34\end{array}$ & $\begin{array}{l}0.32 \\
0.33 \\
0.34 \\
0.35\end{array}$ & $\begin{array}{l}0.82 \\
0.85 \\
0.87 \\
0.91\end{array}$ & $\begin{array}{l}0.45 \\
0.46 \\
0.48 \\
0.51\end{array}$ \\
\hline $\begin{array}{l}319 A-3-4, \\
85-88\end{array}$ & $\begin{array}{l}0.4 \\
1.0 \\
2.0 \\
6.0\end{array}$ & $\begin{array}{l}1.84 \\
1.85 \\
1.86 \\
1.87\end{array}$ & $\begin{array}{l}0.29 \\
0.29 \\
0.30 \\
0.30\end{array}$ & $\begin{array}{l}23.2 \\
24.0 \\
24.6 \\
25.7\end{array}$ & $\begin{array}{l}0.68 \\
0.71 \\
0.73 \\
0.76\end{array}$ & $\begin{array}{l}1.47 \\
1.42 \\
1.38 \\
1.31\end{array}$ & $\begin{array}{l}0.33 \\
0.34 \\
0.34 \\
0.35\end{array}$ & $\begin{array}{l}0.86 \\
0.87 \\
0.89 \\
0.92\end{array}$ & $\begin{array}{l}0.46 \\
0.48 \\
0.50 \\
0.52\end{array}$ \\
\hline $\begin{array}{l}319 \mathrm{~A}-4-1 \\
137-140\end{array}$ & $\begin{array}{l}0.4 \\
1.0 \\
2.0 \\
6.0\end{array}$ & $\begin{array}{l}1.82 \\
1.83 \\
1.83 \\
1.86\end{array}$ & $\begin{array}{l}0.28 \\
0.29 \\
0.29 \\
0.30\end{array}$ & $\begin{array}{l}21.7 \\
22.3 \\
22.7 \\
24.3\end{array}$ & $\begin{array}{l}0.63 \\
0.65 \\
0.66 \\
0.71\end{array}$ & $\begin{array}{l}1.58 \\
1.54 \\
1.51 \\
1.40\end{array}$ & $\begin{array}{l}0.32 \\
0.32 \\
0.33 \\
0.34\end{array}$ & $\begin{array}{l}0.82 \\
0.83 \\
0.85 \\
0.87\end{array}$ & $\begin{array}{l}0.42 \\
0.43 \\
0.44 \\
0.49\end{array}$ \\
\hline $\begin{array}{l}319 A-5-1 \\
80-83\end{array}$ & $\begin{array}{l}0.4 \\
1.0 \\
2.0 \\
6.0\end{array}$ & $\begin{array}{l}1.80 \\
1.81 \\
1.82 \\
1.84\end{array}$ & $\begin{array}{l}0.28 \\
0.28 \\
0.28 \\
0.29\end{array}$ & $\begin{array}{l}23.1 \\
23.8 \\
24.4 \\
25.3\end{array}$ & $\begin{array}{l}0.68 \\
0.70 \\
0.72 \\
0.75\end{array}$ & $\begin{array}{l}1.47 \\
1.42 \\
1.38 \\
1.33\end{array}$ & $\begin{array}{l}0.36 \\
0.36 \\
0.36 \\
0.37\end{array}$ & $\begin{array}{l}0.92 \\
0.93 \\
0.94 \\
0.95\end{array}$ & $\begin{array}{l}0.44 \\
0.46 \\
0.48 \\
0.51\end{array}$ \\
\hline $\begin{array}{l}319 A-6-1 \\
145-148\end{array}$ & $\begin{array}{l}0.4 \\
1.0 \\
2.0 \\
6.0\end{array}$ & $\begin{array}{l}1.86 \\
1.85 \\
1.85 \\
1.87\end{array}$ & $\begin{array}{l}0.30 \\
0.29 \\
0.29 \\
0.30\end{array}$ & $\begin{array}{l}23.1 \\
23.1 \\
23.4 \\
24.1\end{array}$ & $\begin{array}{l}0.66 \\
0.67 \\
0.67 \\
0.70\end{array}$ & $\begin{array}{l}1.50 \\
1.50 \\
1.48 \\
1.43\end{array}$ & $\begin{array}{l}0.31 \\
0.32 \\
0.32 \\
0.33\end{array}$ & $\begin{array}{l}0.81 \\
0.83 \\
0.84 \\
0.85\end{array}$ & $\begin{array}{l}0.46 \\
0.46 \\
0.46 \\
0.48\end{array}$ \\
\hline $\begin{array}{l}319 A-7-1 \\
65-68\end{array}$ & $\begin{array}{l}0.4 \\
1.0 \\
2.0 \\
6.0\end{array}$ & $\begin{array}{l}1.84 \\
1.84 \\
1.84 \\
1.85\end{array}$ & $\begin{array}{l}0.29 \\
0.29 \\
0.29 \\
0.29\end{array}$ & $\begin{array}{l}21.5 \\
22.0 \\
22.0 \\
23.1\end{array}$ & $\begin{array}{l}0.61 \\
0.63 \\
0.64 \\
0.66\end{array}$ & $\begin{array}{l}1.63 \\
1.59 \\
1.57 \\
1.50\end{array}$ & $\begin{array}{l}0.30 \\
0.31 \\
0.31 \\
0.32\end{array}$ & $\begin{array}{l}0.78 \\
0.79 \\
0.80 \\
0.83\end{array}$ & $\begin{array}{l}0.41 \\
0.42 \\
0.43 \\
0.45\end{array}$ \\
\hline $\begin{array}{l}319-13-1, \\
52-55\end{array}$ & $\begin{array}{l}0.4 \\
1.0 \\
2.0 \\
6.0\end{array}$ & $\begin{array}{l}1.87 \\
1.86 \\
1.85 \\
1.87\end{array}$ & $\begin{array}{l}0.30 \\
0.30 \\
0.29 \\
0.30\end{array}$ & $\begin{array}{l}23.6 \\
23.7 \\
24.1 \\
25.6\end{array}$ & $\begin{array}{l}0.69 \\
0.69 \\
0.71 \\
0.75\end{array}$ & $\begin{array}{l}1.45 \\
1.44 \\
1.42 \\
1.33\end{array}$ & $\begin{array}{l}0.32 \\
0.33 \\
0.34 \\
0.35\end{array}$ & $\begin{array}{l}0.82 \\
0.85 \\
0.88 \\
0.91\end{array}$ & $\begin{array}{l}0.48 \\
0.47 \\
0.48 \\
0.52\end{array}$ \\
\hline $\begin{array}{l}320 \mathrm{~B}-3-1 \\
64-67\end{array}$ & $\begin{array}{l}0.4 \\
1.0 \\
2.0 \\
6.0\end{array}$ & $\begin{array}{l}1.84 \\
1.83 \\
1.83 \\
1.86\end{array}$ & $\begin{array}{l}0.29 \\
0.29 \\
0.29 \\
0.30\end{array}$ & $\begin{array}{l}16.8 \\
17.0 \\
17.3 \\
19.3\end{array}$ & $\begin{array}{l}0.46 \\
0.46 \\
0.47 \\
0.53\end{array}$ & $\begin{array}{l}2.18 \\
2.15 \\
2.11 \\
1.88\end{array}$ & $\begin{array}{l}0.22 \\
0.23 \\
0.23 \\
0.25\end{array}$ & $\begin{array}{l}0.58 \\
0.59 \\
0.60 \\
0.64\end{array}$ & $\begin{array}{l}0.31 \\
0.31 \\
0.32 \\
0.37\end{array}$ \\
\hline $\begin{array}{l}\text { 320B-4-1, } \\
144-147\end{array}$ & $\begin{array}{l}0.4 \\
1.0 \\
2.0 \\
6.0\end{array}$ & $\begin{array}{l}1.87 \\
1.86 \\
1.87 \\
1.89\end{array}$ & $\begin{array}{l}0.30 \\
0.30 \\
0.30 \\
0.31\end{array}$ & $\begin{array}{l}19.8 \\
20.5 \\
21.4 \\
23.4\end{array}$ & $\begin{array}{l}0.56 \\
0.58 \\
0.61 \\
0.67\end{array}$ & $\begin{array}{l}1.78 \\
1.72 \\
1.64 \\
1.49\end{array}$ & $\begin{array}{l}0.26 \\
0.27 \\
0.28 \\
0.30\end{array}$ & $\begin{array}{l}0.68 \\
0.71 \\
0.74 \\
0.78\end{array}$ & $\begin{array}{l}0.39 \\
0.40 \\
0.42 \\
0.47\end{array}$ \\
\hline $320 \mathrm{~B}-5, \mathrm{CC}$ & $\begin{array}{l}0.4 \\
1.0 \\
2.0 \\
6.0\end{array}$ & $\begin{array}{l}1.85 \\
1.84 \\
1.82 \\
1.79\end{array}$ & $\begin{array}{l}0.29 \\
0.29 \\
0.28 \\
0.27\end{array}$ & $\begin{array}{l}22.3 \\
22.5 \\
22.4 \\
22.6\end{array}$ & $\begin{array}{l}0.63 \\
0.64 \\
0.64 \\
0.65\end{array}$ & $\begin{array}{l}1.58 \\
1.57 \\
1.57 \\
1.54\end{array}$ & $\begin{array}{l}0.30 \\
0.31 \\
0.32 \\
0.34\end{array}$ & $\begin{array}{l}0.78 \\
0.80 \\
0.83 \\
0.87\end{array}$ & $\begin{array}{l}0.43 \\
0.43 \\
0.42 \\
0.42\end{array}$ \\
\hline $\begin{array}{l}321-13-4 \\
104-107\end{array}$ & $\begin{array}{l}0.4 \\
1.0 \\
2.0 \\
6.0\end{array}$ & $\begin{array}{l}1.84 \\
1.84 \\
1.84 \\
1.87\end{array}$ & $\begin{array}{l}0.29 \\
0.29 \\
0.29 \\
0.30\end{array}$ & $\begin{array}{l}16.8 \\
17.3 \\
17.9 \\
20.0\end{array}$ & $\begin{array}{l}0.47 \\
0.50 \\
0.51 \\
0.57\end{array}$ & $\begin{array}{l}2.11 \\
2.00 \\
1.97 \\
1.76\end{array}$ & $\begin{array}{l}0.23 \\
0.24 \\
0.25 \\
0.26\end{array}$ & $\begin{array}{l}0.59 \\
0.62 \\
0.64 \\
0.68\end{array}$ & $\begin{array}{l}0.32 \\
0.34 \\
0.34 \\
0.34\end{array}$ \\
\hline $\begin{array}{l}321-14-1 \\
76-79\end{array}$ & $\begin{array}{l}0.4 \\
1.0 \\
2.0 \\
6.0\end{array}$ & $\begin{array}{l}1.83 \\
1.82 \\
1.81 \\
1.82\end{array}$ & $\begin{array}{l}0.29 \\
0.28 \\
0.28 \\
0.28\end{array}$ & $\begin{array}{l}22.1 \\
22.1 \\
22.2 \\
23.2\end{array}$ & $\begin{array}{l}0.64 \\
0.64 \\
0.65 \\
0.68\end{array}$ & $\begin{array}{l}1.56 \\
1.55 \\
1.55 \\
1.47\end{array}$ & $\begin{array}{l}0.32 \\
0.32 \\
0.33 \\
0.35\end{array}$ & $\begin{array}{l}0.81 \\
0.83 \\
0.85 \\
0.89\end{array}$ & $\begin{array}{l}0.42 \\
0.43 \\
0.42 \\
0.45\end{array}$ \\
\hline $\begin{array}{l}321-14-4, \\
51-54\end{array}$ & $\begin{array}{l}0.4 \\
1.0 \\
2.0 \\
6.0\end{array}$ & $\begin{array}{l}1.83 \\
1.83 \\
1.82 \\
1.81\end{array}$ & $\begin{array}{l}0.29 \\
0.29 \\
0.28 \\
0.28\end{array}$ & $\begin{array}{l}19.7 \\
20.1 \\
20.5 \\
21.2\end{array}$ & $\begin{array}{l}0.57 \\
0.59 \\
0.60 \\
0.63\end{array}$ & $\begin{array}{l}1.74 \\
1.71 \\
1.67 \\
1.60\end{array}$ & $\begin{array}{l}0.28 \\
0.29 \\
0.30 \\
0.32\end{array}$ & $\begin{array}{l}0.73 \\
0.75 \\
0.77 \\
0.82\end{array}$ & $\begin{array}{l}0.39 \\
0.39 \\
0.40 \\
0.41\end{array}$ \\
\hline
\end{tabular}




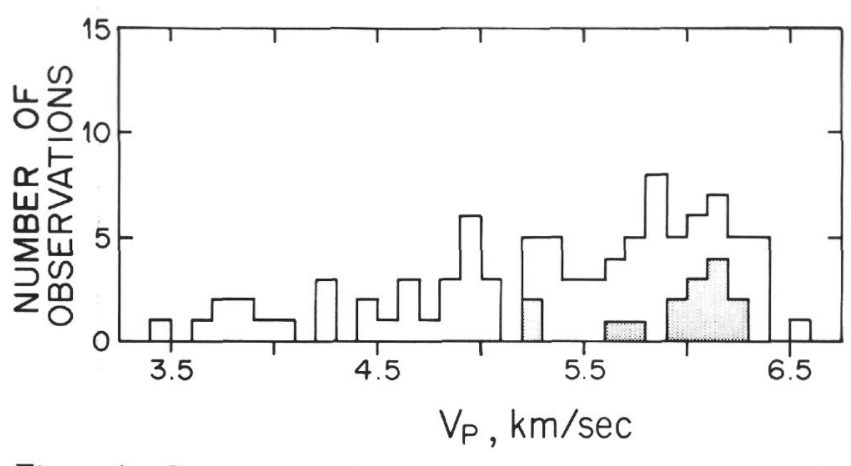

Figure 1. Compressional wave velocities at $0.5 \mathrm{kbar}$ for water-saturated DSDP basalts (Leg 34 velocities shaded). DSDP velocities from Christensen and Salisbury (1972, 1973); Christensen (1973a; 1973b); Christensen et al. (1974a, 1974b).
Christensen, N.I. and Salisbury, M.H., 1972. Sea floor spreading, progressive alteration of Layer 2 basalts, and associated changes in seismic velocities: Earth Planet. Sci. Lett., v. 15 , p. 367.

1973. Velocities, elastic moduli and weathering-age relations for Pacific Layer 2 basalts: Earth Planet. Sci. Lett., v. 19, p. 461.

Christensen, N.I., Fountain, D.M., Carlson, R.L., and Salisbury, M.H., 1974a. Velocities and elastic moduli of volcanic and sedimentary rocks recovered on DSDP Leg 25. In Simpson, E.S.W., Schlich, R., et al., Initial Reports of the Deep Sea Drilling Project, Volume 25: Washington (U.S. Government Printing Office), p. 357.

Christensen, N.I., Salisbury, M.H., Fountain, D.M., and Carlson, R.L., 1974b. Velocities of compressional and shear waves in DSDP Leg 27 basalts. In Veevers, J.J., Heirtzler, J.R., et al., Initial Reports of the Deep Sea Drilling Project, Volume 27: Washington (U.S. Government Printing Office), p. 445. 